\title{
The Hellaz Neutrino Detector
}

\author{
T. Ypsilantis \\ Collège de France, Paris
}

\section{Simulations show that unlike other existing and proposed detectors, a track projection chamber based on 2000 cubic metres of a cold helium - methane gas mixture can help solve the solar neutrino problem by determining the direction and energy of solar neutrinos.}

The most interesting solar neutrinos are pp neutrinos, with a continuum extending up to an energy of $420 \mathrm{keV}$, and the monoenergetic $862 \mathrm{keV}$ 'Be neutrinos formed in the $\mathrm{p}+\mathrm{p} \rightarrow \mathrm{e}+\mathrm{d}+v_{\mathrm{e}}$ and $\mathrm{e}+{ }^{ } \mathrm{Be}$ $\rightarrow{ }^{\top} \mathrm{Li}+v_{\mathrm{e}}$ reactions. All neutrinos have a known flavour (electron-neutrino $v_{\mathrm{e}}$ ) at the sun and energy production in the sun mostly transits through the first reaction, so the pp neutrino flux is essentially determined by the solar luminosity. Since the neutrino-electron (ve) elastic scattering cross-section is given by the well tested electroweak theory, any observed differences in flux, flavour or spectral shape with respect to theoretical predictions will require either new physics (such as flavour oscillations whereby one type of neutrino mixes into another type) or a major revamping of the Standard Solar Model that describes the Sun's nuclear physics and hydrodynamics. This is the so-called the solar neutrino problem (see insert for a discussion of the latest situation).

A new solar neutrino experiment called Hellaz (Helium at liquid azote nitrogen - temperature) is being proposed to help resolve the problem using pp solar neutrino data. It consists of 6 tonnes of cold helium gas as a target for neutrinoelectron elastic scatters. A recoil electron from a ve event leaves a trail of ionization electrons which define a track. Each ionization electron drifts in an applied axial electric field until it reaches a twodimensional $x, y$-detector located at each end of a cylindrical target volume (Fig. 1). Following the track projection chamber (TPC) method, the $z$-coordinate is obtained from the electron arrival time. The $x, y, z$ data determine the recoil electron energy and direction and, given the sun's position, the neutrino energy $E_{v}$.

Tom Ypsilantis, a research physicist in high-energy particle physics based at the Collège de France in Paris, is currently working on neutrino experiments (Hellaz and Long Baseline $\mathrm{RICH}$ ) and on applications of the $\mathrm{RICH}$ technique to $\mathrm{B}$-physics at CERN's future LHC collider. He has worked at the Ecole Polytechique, Paris, the CE Saclay, CERN, and the University of Southern California, Berkeley.
Hellaz will measure recoil electrons with a kinetic energy $T$ as low as $100 \mathrm{keV}$ corresponding to a range of about $50 \mathrm{~mm}$ because it is filled with gases of low atomic number and low density. Simulations demonstrate that $T$ is measured to within $3 \%$ and the electron direction to within 35 $\mathrm{mrad}$ so the energy of incoming $300 \mathrm{keV}$ neutrinos can be determined to within 2 to $4 \%$ - an advantage of Hellaz in contrast to existing and proposed $v e$ detectors (see insert). The proposed Borexino can detect recoil electrons with energies above 260 $\mathrm{keV}$, but cannot determine the electron direction, and hence the neutrino energy.

Moreover, the cross-sections $\sigma$ and differential cross-sections $\mathrm{d} \sigma / \mathrm{d} T$ for $v_{\mathrm{e}} \mathrm{e}$ electron-neutrino and $v_{\mu}$ e gammaneutrino elastic scattering on electrons are sufficiently different to allow a sensitive determination of the flavour and flux of incoming neutrinos independently of solar models or mixing scenarios. Indeed, Hellaz will be able to determine the amount of flavour mixing for $\mathrm{pp}$ and pep neutrinos to permit resolution of all the current neutrino mixing scenarios.

Hellaz is also competitive when compared to other much heavier detectors. The calculated rate of ve elastic events from the $\mathrm{pp}$ and ${ }^{7} \mathrm{Be}$ solar neutrino fluxes is about 16 events/day for 6 tonnes of helium gas if all recoil electrons ( $T \geq 0$ $\mathrm{keV})$ are detected. With the more realistic limit of $T \geq 100 \mathrm{keV}$ there will still be 11 events/day. By comparison, the Gran Sasso Underground Laboratory's $\mathrm{GaCl}_{3}$-based GALLEX detector with the same threshold and twice the mass only detects 0.2 events/day, since although the crosssection of ${ }^{71} \mathrm{Ga}$ is 4.5 times the average ve cross-section, its nuclear density is only $1 / 88$ of the helium electron density.

Several prototypes of the Hellaz detector have been constructed and are now under test. A $40 \mathrm{~m}^{3}$ device will soon be placed in a tunnel at Gran Sasso with full carbon dioxide (or $\mathrm{B}_{2} \mathrm{O}_{3}$ ) shielding to confirm estimates of the radiopurity of materials by neutron activation analysis and to measure the ve signal and the gammaelectron ( $\gamma$ e) background. It will also serve an ideal tool for searching for dark matter, an aspect that will not be discussed.

\section{Direction and Energy Resolution}

The precision with which the direction and energy of the incident neutrinos can be determined in Hellaz has been estimated using accurate simulations of

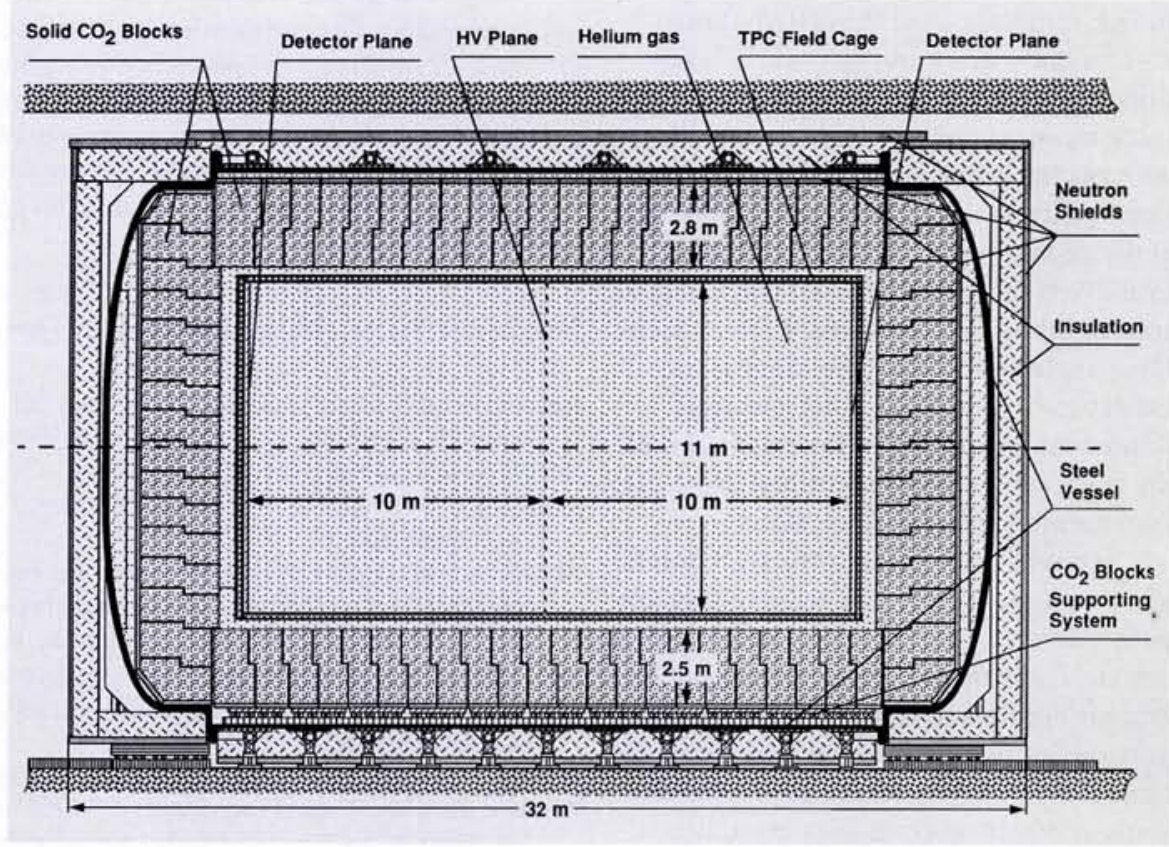

Fig. 1. A longitudinal view of the heavily shielded, cylindrical Hellaz time proportional counter solar-neutrino detector. Ionization tracks drift in an axial field generated by a field cage to modular multiwire detectors located at the endcaps which use the same low-temperature helium-methane gas mixture as the target volume. 
ionization in helium gas. A typical path in the $z$-direction for a recoil electron with a kinetic energy of $100 \mathrm{keV}$ is shown in Fig. 2. The change of the path direction is only caused by multiple scattering since drift diffusion is not included. The simulations show that the mean deflection in the first $10 \mathrm{~mm}$ is about $1 \mathrm{~mm}$ so a direction error $\sigma_{\Theta}$ of less than 100 mrad may be expected if the sampling is sufficient. Owing to the build-up of multiple scattering, the length of the sampled track length is limited to about $20 \mathrm{~mm}$, and the direction error is estimated to be $43 \mathrm{mrad}$.

The average error caused by diffusion on allowing the electrons to drift to $\mathrm{Hel}$ laz's multiwire chamber detectors is $3 \mathrm{~mm}$ for a maximum drift length of $10 \mathrm{~m}$. However, the detector will use time bins of 20 ns corresponding to a longitudinal diffusion drift distance $\Delta z$ of only $0.014 \mathrm{~mm}$. This is to ensure single-electron sampling: each electron will effectively arrive separately and be seen efficiently by the detector. On average, about $50 \%$ of the electrons arrive singly and give unambiguous $x, y, z$ coordinates for tracking (the remaining $50 \%$ appear as doubles, triples, etc.).

The principal axes of the electron cloud that makes up a track are determined by diagonalizing the track's moment of inertia tensor in order to fit a straight line. The calculated distribution, projected onto the three principle axes, of hits on the multiwire chamber detector for a track made up of three recoil electrons with $3 \mathrm{~mm}$ drift diffusion added is shown in Fig. 3. As expected, the distributions along $y$ and $z$ are symmetric with threedimensional Gaussian smearing of the track by an amount $\sigma_{y} \approx \sigma_{z} \approx 3 \mathrm{~mm}$ whereas the $x$-distribution shows the range of the track and increased ionization at the end of the track. This particular example is for a well-behaved track in contrast to other tracks that show the effect of large angle deflections (the range is too small for the ionization). But the most important feature is that the start point of the track can be easily identified using the transformed coordinate system.

Straight lines through the start points of $s=12 \mathrm{~mm}, T=100 \mathrm{keV}$ tracks for pixels with a size $\Delta x=1 \mathrm{~mm}, \Delta z=0.014 \mathrm{~mm}$ and 428 electrons show that the $x, z$-projection error is dominated by the diffusion error, and that increasing the length of the track from 12 to $22 \mathrm{~mm}$ reduces the projection error. A direction error of $35 \mathrm{mrad}$ is obtained by fitting data to a theoretical curve and then quadratically combining the $x, z$ - and $y, z$-projection errors.

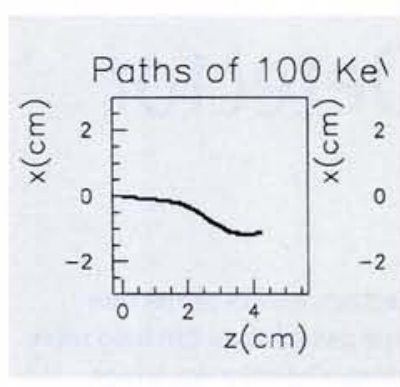

Fig. 2. The path, in the $z$-direction, of simulated $100 \mathrm{keV}$ electron recoil in helium gas at $77 \mathrm{~K}$ and 5 bar without drift diffusion; the observed curvature is due to multiple scattering.
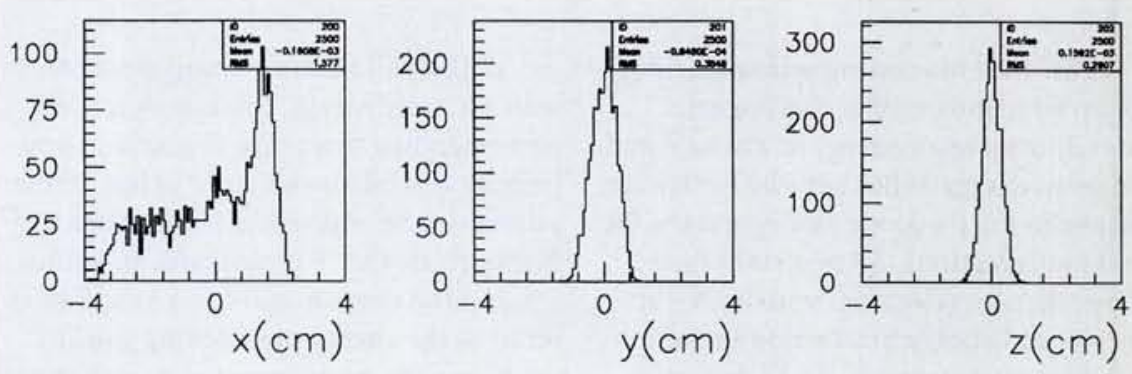

Fig. 3. The simulated distributions of the number of hits on the multiwire chamber detector for three tracks with $3 \mathrm{~mm}$ drift diffusion added. The distributions, obtained by transforming the coordinate system of the cloud of recoil electrons that make up a track, are projected on the principal axes of the track.

\section{The Solar Neutrino Problem Today}

The solar neutrino problem arises because theorists have constructed an evolutionary model of the sun based on the premises that it is spherical, in hydrostatic equilibrium with energy transport via photon radiation and conversion, and involves thermonuclear burning of the light elements as the energy source. The input parameters are the sun's mass, radius, elemental abundances, and nuclear reaction rates. The initial $\mathrm{He}$ and $\mathrm{H}$ abundances are adjusted so that the model gives, after a time evolution of $4.65 \times 10^{9}$ years, i.e., the sun's age, the presently known solar luminosity and temperature. The model therefore predicts fluxes of neutrinos from each of the contributing nuclear reactions (the pp continuum, the two monoenergetic ${ }^{7} \mathrm{Be}$ lines, the monoenergetic pep line, and the ${ }^{8} \mathrm{~B},{ }^{13} \mathrm{Ne},{ }^{15} \mathrm{O}$, and ${ }^{17} \mathrm{~F}$ continua). It also predicts a myriad of acoustic oscillation frequencies. These have been measured to high precision and now agree to within about $0.2 \%$. So in a sense the model is confirmed.

This is not the case for neutrinos: the observed fluxes are $30-50 \%$ of model predictions. Analyses of data indicate that the ${ }^{7} \mathrm{Be}$ flux is very low or zero whereas the ${ }^{8} \mathrm{~B}$ is $50 \%$ of expectations. This is of course impossible because the production of ${ }^{8} \mathrm{~B}$ proceeds through ${ }^{7} \mathrm{Be}$. In addition, the two experiments which measure the pp neutrinos (GALLEX and SAGE) see only a minimum flux consistent with the pp reaction (largely fixed by solar luminosity), leaving little for the other channels. So the measured neutrino fluxes are inconsistent with the solar model implying that the model is wrong. An alternative explanation requires new particle physics. If neutrinos can oscillate between $v_{e}, v_{\mu}$ and $v_{\tau}$ then the data is consistent with the model because an inverse $\beta$-decay detector sees only the $v_{e}$ 's. Next-generation detectors will hopefully resolve this difficulty because they are sensitive to some degree to all neutrino flavours.

\section{Underground Solar Neutrino Detectors}

Homestake (USA): $615 \mathrm{t}_{2} \mathrm{Cl}_{6}$ liquid $\left(127 \mathrm{t}^{37} \mathrm{Cl}\right) ; \mathrm{v}_{\mathrm{e}}+{ }^{37} \mathrm{Cl} \rightarrow{ }^{37} \mathrm{~A}+\mathrm{e}$ reaction observed by detection of ${ }^{37} \mathrm{~A}$ radioactivity; $814 \mathrm{keV}$ reaction threshold; sensitive to ${ }^{7} \mathrm{Be}$ but not to pp neutrinos; 0.07 events/day.

GALLEX (Gran Sasso, Italy): $12 \mathrm{t}^{71} \mathrm{Ga}$ in $77 \mathrm{t} \mathrm{GaCl}_{3} ; v_{\mathrm{e}}+{ }^{71} \mathrm{Ga} \rightarrow{ }^{71} \mathrm{Ge}+\mathrm{e}^{-}$reaction; 0.2 events/day.

SAGE (Russia): $22 \mathrm{t}^{71} \mathrm{Ga}$ in $55 \mathrm{t}$ of Ga metal; threshold sufficiently low ( $233 \mathrm{keV}$ ) to detect pp and ${ }^{7}$ Be neutrinos; $\approx 0.1$ events/day.

Kamiokande III (Japan): only existing real-time neutrino detector; fiducial volume of 680 t of water; electron recoils detected via Cherenkov light emission but owing to dissolved radioactivity, electron threshold energy $T$ must be set high ( $\geq 7.5 \mathrm{MeV}$ ), hence the low rate; not sensitive to $\mathrm{pp}$ neutrinos; $0.3 /$ day.

SNO (Canada); Super Kamiokande (Japan): soon to be operational; real-time, Cherenkov detectors; electron threshold $T \geq 5 \mathrm{MeV}$; high-energy ${ }^{8} \mathrm{~B}$ neutrinos. SNO: 1 kt $\mathrm{D}_{2} 0$ target; 2 events/day; Super Kamiokande: $22 \mathrm{kt}$ $\mathrm{H}_{2} 0 ; 44$ events/day.

ICARUS (Gran Sasso): proposed real-time ionization detector; $5 \mathrm{kt}$ liquid Ar; 9 ve elastic events/day for $T \geq 5 \mathrm{MeV}$.

Borexino (Gran Sasso): $100 \mathrm{t}$ liquid scintillator; $T \geq 260 \mathrm{keV}$; sensitive to the ${ }^{7} B e$ but not to pp neutrinos; can detect electrons with $T \geq 260 \mathrm{keV}$ but cannot determine electron direction, hence $E_{v}$. More important than ICARUS because of its low threshold; 30 ve events/day.

Hellaz (proposed): $6 \mathrm{t}$ He gas mixture $\left(2000 \mathrm{~m}^{3}, 5\right.$ bar, $\left.77 \mathrm{~K}\right)$; competitive because it detects pp and ${ }^{7}$ Be neutrinos; 11 events/day. 
The track energy is determined either from single-electron counting or from the range of the track after the principle-axis transformation. A direction error of 35 mrad and an electron energy resolution $\sigma_{T} / T$ of $2-4 \%$ measured by single-electron counting gives the neutrino energy resolution, which is plotted as a function of the neutrino energy in Fig. 4 for fixed T. The data show that the neutrino energy resolution is $3-7 \%$ in the $220-420 \mathrm{keV}$ energy range of pp solar neutrinos.

\section{Background Subtraction}

Hellaz is immune to many backgrounds because of the excellent pattern recognition properties of the detector and the extreme purity of the detection medium. Solar neutrinos are not energetic enough to interact inelastically with ${ }^{4} \mathrm{He}$ nuclei so the only process available is ve elastic scattering. The dominant background is ve Compton scattering with the same kinematics as the ve signal but with a cross-section $10^{20}$-times larger.

The tracking and energy capabilities of Hellaz allow rejection rates of up to $10^{4}$ gammas/day by identifying a large sample of ve Compton electron recoils which cannot be due to solar neutrinos because the calculated energy neutrino energy is negative or the event is scattered twice. Using these events in delayed (or random) coincidence with the sun $S$ allows one to trace the background $B$ under the pp continuum. Simulations for a background rate of $10^{4}$ gammas/day (33-times the expected rate for external and internal backgrounds) show that the detected signal $S_{\mathrm{d}}$ is $S+B$ with only 126 gammas/day are under the pp continuum. Because the background is measured so well, no additional statistical error accrues in the difference $S_{\mathrm{d}}-B$, which therefore gives the true solar signal $S$.

An additional background source is from $\beta$-decay of ${ }^{14} \mathrm{C}$ from the detector's methane. This is minimized by using methane that is older than $10^{6}$ years, but it cannot be completely eliminated. For methane of this age the remaining ${ }^{14} \mathrm{C}$ activity is due to the ${ }^{13} \mathrm{C}(\mathrm{n}, \gamma){ }^{14} \mathrm{C}$ reaction, with neutrons coming from $(\alpha, \mathrm{n})$ reactions in rock. Based on measurements made using the Borexino detector, the methane background is about 10-times larger than that from the detector materials and can be subtracted. Thus ${ }^{14} \mathrm{C}$ activity will not dominate the experiment. Nevertheless, quenching gases which do not contain carbon are being investagated in order to replace methane.

Fig. 4. The calculated neutrino energy resolution $\sigma_{E_{v}} / E_{v}$ as a function of the neutrino energy $E_{y}$ for various values of the kinetic energy $T$ of the recoil electron assuming the counting of single electrons by the Hellaz multiwire chamber detector.

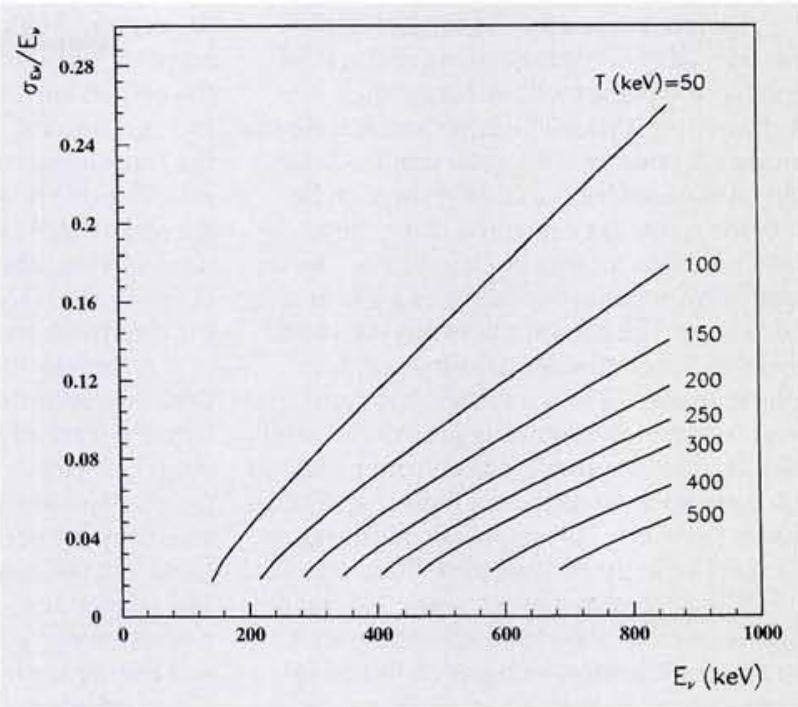

\section{Flux and Mixing}

It can be shown that the differential cross-sections for $\mathrm{e} v_{\mathrm{e}}$ and $\mathrm{e} v_{\mathrm{I}}$ interactions as a function of the electron energy depend only on the neutrino flavour mixing ratio $f$ provided the neutrino energy is not large compared to one-half the electron mass (i.e., $E_{v} \approx m_{\mathrm{e}} / 2$ ). Integration then gives the counts $n_{i}$ in the $i$ th energy bin of width $w$ and the total number of counts $n_{\text {tot }}$ in $p$ bins as

$$
\begin{aligned}
& n_{i}=K \Phi w\left(a_{i}-b_{f} f\right) \\
& n_{\text {tot }}=K \Phi w p(d-e f)
\end{aligned}
$$

where $K=n_{e}\left(\sigma_{o} / m_{\mathrm{e}}\right) \Delta t$ for $n_{\mathrm{e}}$ target electrons and an exposure time $\Delta t ; a_{i}, b_{i}, d$, and $e$ are coefficients known from electroweak theory; $\Phi$ is the initial flux of $v_{\mathrm{e}}$ neutrinos at the Sun and $\Phi(1-f)$ and $\Phi f$ are the $v_{\mathrm{e}}$ and $v_{\mu}$ fluxes, respectively, on Earth. Eq. (2) gives $f=d\left(1-n_{\text {tot }} / n_{\mathrm{o}}\right) / e$ where
$n_{\mathrm{o}}=K \Phi p w d$ is the number of events expected for $f=0$ so a measurement of $n_{\text {tot }}$ suffices to determine $f$ if the flux $\Phi$ is known. This is the case for the pp solar neutrinos because $\Phi_{\mathrm{pp}}$ is fixed by the observed solar luminosity. A 5-year Hellaz run will determine $f$ to $1-3 \%$.

The flux $\Phi$ may be eliminated from (1) and (2) to show that $\left.n_{i} p /<n_{\text {tot }}\right\rangle$, the normalised number of counts in a bin, depends only on $f$, which can be determined from the data without reference to solar models. Knowing $f$ one obtains the absolute flux $\Phi$ from Eq. (1). This independent determination of $(f, \Phi)$ works for pp and pep neutrinos (but not for ${ }^{7} \mathrm{Be}$ ) because it requires $E_{v} \approx m_{\mathrm{e}} / 2$. The observation of $f \neq 0$ would of course constitute evidence for neutrino oscillations since only electron-neutrinos are produced in solar nuclear reactions, and Hellaz will have helped solve the solar neutrino problem.

\section{The Hellaz Detector}

Hellaz consists of 6 tonnes, approximately $2000 \mathrm{~m}^{3}$, of a helium gas mixture at 5 bar and $77 \mathrm{~K}$. The gas serves as a target for neutrino-electron (ve) elastic scattering events because a recoil electron from an event leaves a trail of ionization electrons (and photo-electrons - see below) which defines a track. A uniform axial electric field parallel to the longitudinal $z$-axis of the detector volume causes the electrons to drift to two-dimensional $x, y$ ionization detectors located at each end of the $20 \mathrm{~m}$ long cylindrical volume. The track projection chamber (TPC) method gives the $z$-coordinate from the electron arrival time to allow the electron track locus to be measured.

Hellaz uses multiwire chamber (MWC) detectors because their high gain and high sensitivity for single electrons provide the copious sampling needed to determine the track direction and to identify the head and tail of a track, and hence its range and energy. By operating the ionization detectors in the same high purity helium - $10 \%$ methane (by volume) drift gas mixture (obtained by freezing out impurities at $4 \mathrm{~K}$ ) as the target, and using sufficient gain to resolve a significant number of single electrons, typical electron lifetimes are greater than $200 \mathrm{~ms}$. The lifetimes therefore exceed the maximum drift time of $\approx 10 \mathrm{~ms}$ by a large margin. Single-electron sampling also endows the detector with a calorimetric capability which gives a precise determination of the kinetic energy of the recoil electrons (as compared to their range). 
Each $100 \mathrm{~m}^{2}$ endcap is composed of modular MWCs made of $10 \mathrm{~mm}$ thick Kevlar honeycomb planes with $100 \mathrm{~mm}$ thick Kevlar-epoxy skins. The inner surface of each module is covered with a $100 \mathrm{~mm}$ thick Kapton layer on which $0.95 \mathrm{~mm}$ wide metallic cathode strips are deposited along the $x$ direction with a $1 \mathrm{~mm}$ pitch in $y$. The $1 \mathrm{~m}$ long MWC wires are strung along y $(1 \mathrm{~mm}$ pitch in $x$ ). The distance between the anode wires and the cathode strips is fixed by quartz spacers. The wires and strips are made up into economically priced $1 \mathrm{~m}^{2}$ modules in order to provide the enormous area of the detector $\left(200 \mathrm{~m}^{2}\right)$ and a high spatial resolution $(\approx 1 \mathrm{~mm})$. This imposes conditions on the drift velocity and electron diffusion.

The approximately 2.5 tonne TPC field cage is made of 200 mm thick radiopure Kevlar or acrylic honeycomb panels joined to form a decahexahedron; $20 \mathrm{~mm}$ pitch metallic field shaping strips are deposited on the surface. A radiopure metal mesh, built into the middle of the cylindrical field cage, provides the $200 \mathrm{kV}$ equipotential plane needed to produce the electric drift field.

The energy resolution of a MWC is usually dominated by fluctuations in amplification. If the MWC has a good single-electron sensitivity then only ionization fluctuations are important. However, energy loss in helium gas is a special case because the dominant energy loss processes are ionization and scintillation, with very little energy lost to excitons. Methane has a large photoabsorption cross-section, so methane added to the helium ensures that photoelectrons injected into the TPC gas mixture very near the track locus convert a large fraction of the scintillation energy back into ionization. Single counting of all of the 2500 ionization electrons and the 1000 photoelectrons produced in the track gives a statistical error for the electron kinetic energy $T$ of about $1.5 \%$.

A time-to-digital-converter with $20 \mathrm{~ns}$ time bins records $1.3 \mathrm{~ms}$ of drift time, equivalent to a track of less than $1.3 \mathrm{~m}$ in length at $T$ $\approx 850 \mathrm{keV}$ keV. A starting trigger is obtained from a coincidence of contiguous wires and/or strips occurring when the first electrons of a track arrive at the detector plane. The trigger does not need to determine the $z$-position of a track since the track position and energy are found by fitting lines to transformed tracks.

To avoid ambiguities in identifying $x, y$ coordinates it is essential that electrons arrive singly in the $20 \mathrm{~ns}$ time bins. This requires a gas with a slow drift velocity $(\approx 1$ $\mathrm{mm} / \mathrm{ms}$ ). Obtaining the necessary angular precision requires a gas with low diffusion coefficient. A methane -10 volume $\%$ methane gas mixture satisfies both criteria.

A critical feature is the shield needed to absorb $\gamma$-rays and neutrons from the walls of the cavern deep underground in which the detector is placed. Blocks of solidified carbon dioxide can be used because they are radio- pure, inexpensive, strong, cold, and dense. A low temperature $(77 \mathrm{~K})$ shield is useful because it condenses radon which could otherwise diffuse into the TPC gas. The shield could also be made of bismuth oxide because the commercial product is relatively radiopure and can be further refined. It is, in addition, cheaper and denser than carbon dioxide, thus saving space in cavern, and one could assemble the massive shield at room temperature.

The $\gamma$-ray and neutron fluxes incident on the Hellaz shield are due mainly to ${ }^{238} \mathrm{U}$, ${ }^{232} \mathrm{Th}$ and ${ }^{\circ} \mathrm{K}$ in Gran Sasso rock. Absorption in an early version of the shield has been simulated to investigate the efficiency of carbon dioxide to attenuate gammas and neutrons from the rock and the steel pressure vessel. The calculated final rate into the TPC gas was $<1$ event/day so the shield can suppress essentially all the external gammas and neutrons if it is made and assembled under hermetic conditions.

Since the TCP field cage cannot be shielded it must made of intrinsically low activity material. Assuming that today's level of purity for acrylic can also be attained in Kevlar (otherwise acrylic will be used), the 2.6 tonne TPC cage would produce about 200 decay chains per day each yielding $1.5 \mathrm{gam}$ mas at an energy above $217 \mathrm{keV}$, the minimum to produce a $T \geq 100 \mathrm{keV}$ electron. So 300 gammas/day are expected from the TPC cage.

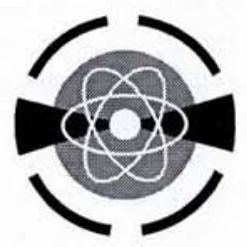

\section{EC Training \& Mobility of Researchers Programme Access to Large-Scale Facility Activity CALL FOR PROPOSALS}

\section{CEA-LV \\ Centre D'Etudes de Limeil-Valenton}

The Centre d'Etudes de Limeil-Valenton of the French Commissariat à L'Energie Atomique holds two of Europe's highest power glass neodymium laser facilities: Phebus and P102. Both are operated in favour of a research programme concentrated on basic plasma physics and Inertial Confinement Fusion.

Phebus is a two-beams system which can be operated either at $0.53 \mu \mathrm{m}$ or $0.35 \mu \mathrm{m}$ with routine characteristics $\sim 6 \mathrm{KJ}-1 \mathrm{~ns}$. A third beam $(\sim 1 \mathrm{KJ})$ is used for plasma probing purposes. More than thirty plasma diagnostics are available on the target chamber, mostly devoted to X-ray analysis with high spatial and temporal resolutions.

\section{P102 works in the sub-picosecond range and provides irradiances greater than $10^{19} \mathrm{~W} / \mathrm{cm}^{2}$.}

Researchers throughout the Member States of the Community and the Associated States (Iceland, Liechtenstein and Norway) who wish to have access to CEA-LV facilities during the next three years (July 1996 - July 1999) are invited to apply.

Proposals will be selected on the basis of scientific merit through an independant peer review procedure. Access will be provided free of charge and will include all infrastructural, logistical, technical and scientific support. Travel and subsistence expenses will be reimbursed.

Contact M. Decroisette for further details and application.

E-mail: decroise@limeil.cea.fr

Tel.: (1)45956362

Fax: (1)43867426 